\title{
Extended measurement setup for transient TEM waveguide characterization
}

\author{
Niklas Briest ${ }^{1}$, Heyno Garbe ${ }^{1}$, and Stefan Potthast ${ }^{2}$ \\ ${ }^{1}$ Institute of Electrical Engineering and Measurement Technology, Leibniz Universität Hannover, Hannover, Germany \\ ${ }^{2}$ Bundeswehr Research Institute for Protective Technologies, NBC-Protection, Munster, Germany \\ Correspondence to: Niklas Briest (briest@geml.uni-hannover.de)
}

Received: 12 January 2016 - Revised: 18 April 2016 - Accepted: 26 June 2016 - Published: 28 September 2016

\begin{abstract}
This paper discusses a field measurement method, based on a two-antenna setup, to qualify the transmission of transient signals inside a GTEM cell. The transmission characteristic of the GTEM1250 is evaluated by the Pearson correlation coefficient (PCC) and is presented with a heatmap. Due to deviations of the uncertainty contribution of the field homogeneity, the frequency band around $100 \mathrm{MHz}$ is evaluated and its effect to the PCC is discussed. Therefore, a comparable narrowband transient signal, a damped sinusoidal (DS) is used. Furthermore, a detailed discussion focussing on nonlinear and distorting effects of the GTEM1250 is performed. The measurements in time domain (TD) identify comparable high secondary $E$-field components in the propagation direction, which are characteristic for higher order modes. Based on the same setup, another measurement is performed in frequency domain (FD) and relates the phase response of the GTEM cell to the above mentioned effects. According to the measured phase response the propagation time is discussed to investigate the distorting effects caused by higher order modes.
\end{abstract}

\section{Introduction}

The interconnection of electronic systems is very high and a continuous functionality is essential. Therefore emission and immunity measurements of all parts of a system is required in order to prevent from EMI (Electromagnetic Interference) effects. Those measurements, especially high-altitude electromagnetic pulse (HEMP) immunity tests, can be performed in transverse electromagnetic (TEM) waveguides (Radasky et al., 1996). The corresponding standardized measurement procedure is defined in IEC 61000-4-20 Annex C and is based on a reference pulse with a double exponential waveform. Due to the wide frequency spectrum of this reference pulse, a characterization and validation of TEM waveguides is performed in time domain (TD). There a TEM waveguide is supplied with the above mentioned pulse. The transmission quality of the TEM waveguide is evaluated by a parameter comparison - the rise time and the pulse width - between the defined reference pulse, which is supplied to the waveguide, and a measured waveform in the test volume.

It should be discussed if the transmission quality of a TEM waveguide for any arbitrary transient signal, whose frequency spectrum is comparable to that of the reference pulse, can be sufficiently described by this reference pulse in TD. Hence, in this paper, another field measurement method to verify the transmission quality of transient signals in GTEM waveguides is presented. The method is based on a two antenna measurement, extended to Kölling et al. (2011), and evaluates the transmission quality based on a correlation between those two measured signals. For the wideband excitation, a damped sinusoidal (DS) waveform is used. Therefore a center frequency of $100 \mathrm{MHz}$ is used. Hamann and Garbe (2014) investigated the GTEM1250 in the frequency domain (FD) and calculated a high uncertainty contribution of the $E$-field homogeneity at $100 \mathrm{MHz}$, which represents a critical frequency of the GTEM1250. Furthermore the TEMmode conditions are investigated in TD and FD, to discuss this critical frequency in detail. Thereby an analysis of the secondary $E$-field components is performed in TD and the propagation time is calculated on basis of the measured phase response in FD. 


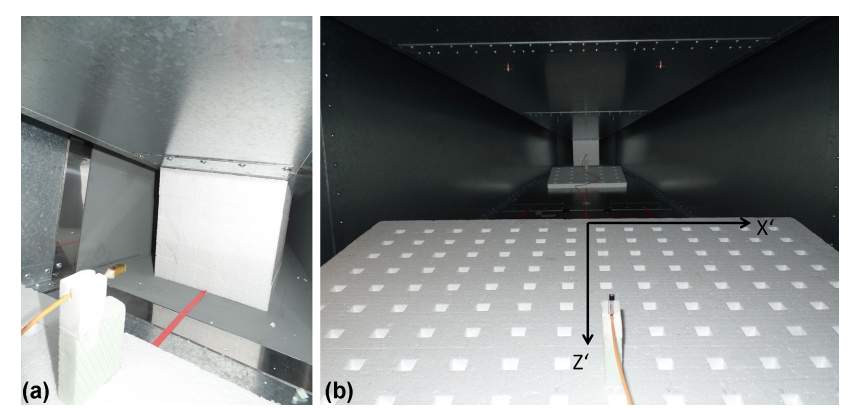

Figure 1. Two antenna measurement setup. (a) Probe at reference position. (b) View from the absorber structure to the test volume.

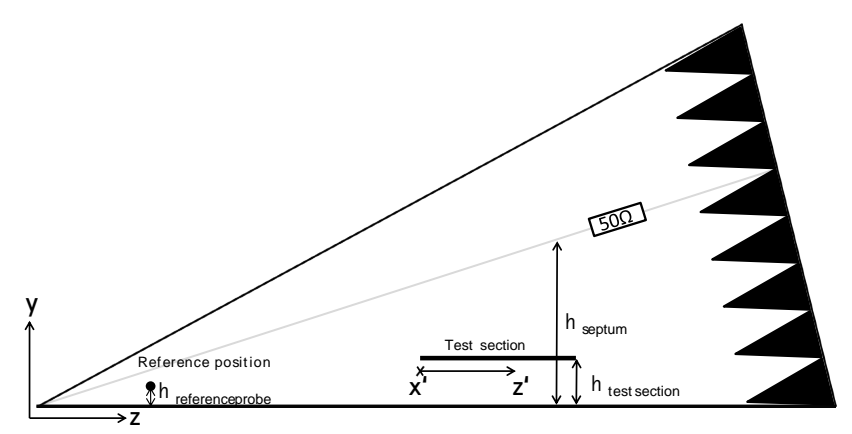

Figure 2. Sketched side view of the GTEM cell, including the position of reference and test section.

\section{Test setup}

The transmission quality of the TEM waveguide is evaluated on basis of the correlation between a reference signal and a signal measured within the test volume. Therefore two measuring positions are defined, which are depicted in Fig. 1 and sketched in Fig. 2.

The first position (Fig. 1a) is located at a septum height of $130 \mathrm{~mm}$, centred to the septum. At this position it is assumed, that no higher order modes are capable to propagate and the field consists of a pure TEM-mode, as explained by Koch (1998). Thus, the measured $E$-field at this position can be used as the reference signal. The test volume of the GTEM1250 - the second measuring position - is depicted in Fig. 1b. According to the manufacturers recommendations (TESEQ, 2014), the measurement positions are placed in a height of $0.541 \mathrm{~m}$ above the bottom of the cell, realized by a board of foamed polystyrene. The test volume is sampled in 64 measuring positions, which can be seen as cavities in Fig. 1b. For the test volume a separated coordinate system is included (Fig. 1b). In each direction, $x^{\prime}$ and $z^{\prime}$, the cavities have a distance of $130 \mathrm{~mm}$ to each other.

At the reference position and the measuring positions in the test volume two identical optical $E$-field probes (Krotke and Mann, 2009) are used, which are mounted by a foamed polystyrene bracket (Fig. 1). Performing this two antenna measurement setup with two identical probes, no antenna factor has to be taken into account and the measured waveforms can be compared directly. Furthermore, measuring the $E$-field signals at the described positions simultaneously, reduces the influences of the signal generator reproducibility, which is a major advantage in comparison to the method described by Kölling et al. (2011) and Briest ey al. (2015).

To evaluate the transmission characteristics of the GTEM cell, the PCC between the reference signal and the signals measured at each position within the test volume is calculated according to Kölling et al. (2011) and is adapted to the present measuring setup in Eq. (1).

$$
\begin{aligned}
& \rho\left(E_{y, \mathrm{r}}, E_{y, \mathrm{~s}}\right)= \\
& \frac{\frac{1}{N} \sum_{k=1}^{N}\left(E_{y, \mathrm{r}}[k]-\bar{E}_{y, \mathrm{r}}\right)\left(E_{y, \mathrm{~s}}[k]-\bar{E}_{y, \mathrm{~s}}\right)}{\sqrt{\frac{1}{N} \sum_{k=1}^{N}\left(E_{y, \mathrm{r}}[k]-\bar{E}_{y, \mathrm{r}}\right)^{2}} \times \sqrt{\frac{1}{N} \sum_{k=1}^{N}\left(E_{y, \mathrm{~s}}[k]-\bar{E}_{y, \mathrm{~s}}\right)^{2}}}
\end{aligned}
$$

The primary $E$-field component $E_{y}$ at the reference position is represented by $E_{y, \mathrm{r}}$ and by $E_{y, \mathrm{~s}}$ within the test volume. The calculated PCC can be analysed by means of a heatmap, where at each position within the test volume $\rho\left(E_{y, \mathrm{r}}, E_{y, \mathrm{~s}}\right)$ is represented and the transmission characteristic of the GTEM1250 can be evaluated.

In addition to this evaluation method and considering, that a DS with a center frequency of $100 \mathrm{MHz}$ as a critical frequency of the GTEM1250 is used, further investigations with a focus on distorting effects and a detailed discussion of the TEM-mode are presented. The pure TEM-mode can be identified on basis of three parameters (Balanis, 2005):

- the ration of the measured $E$ and $H$ field $\frac{E}{H}=120 \pi \Omega$

- the group velocity of the TEM wave $v=c_{0}$

- the components $E$ and $H$ of the TEM wave are orientated perpendicular to each other, what can be investigated by measuring the secondary field components

On basis of the presented measurement setup two criteria can be proofed, the existence of secondary $E$-field components $\left(E_{x}\right.$ and $\left.E_{z}\right)$ and the group velocity respectively the propagation time of the TEM wave, which can be calculated from the phase response measured in FD.

The secondary $E$-field components have to be measured at the reference position and within the test volume. Hence, the assumption that the pure TEM-mode exists at the reference position and possible deviations from the TEM-mode within the test volume can be verified. Therefore the polarization of the probe is changed. It is alternated into the polarizations of $E_{x}$ and $E_{z}$. The secondary components at both positions can be compared and the relation to the primary component $E_{y}$ can be discussed, to identify distorting influences and if the pure TEM-mode is present.

Further the group velocity is a parameter which describes the TEM-mode. A pure TEM wave propagates with the vac- 


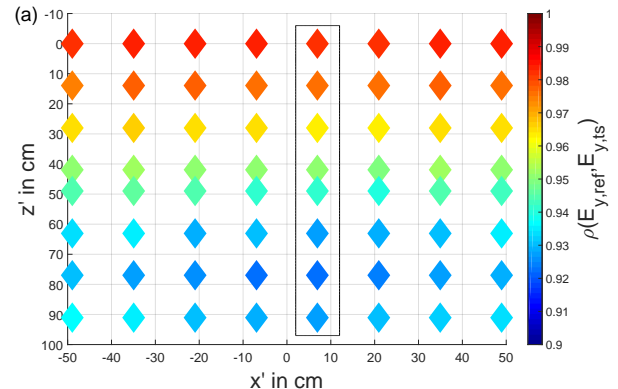

Figure 3. GTEM1250 transmission characteristic evaluated by PCC. $x^{\prime}=7 \mathrm{~cm}$, along the $z^{\prime}$ axis.

uum speed of light $c_{0}$. The propagation speed can be estimated by the measured phase response of the TEM waveguide in FD. Therefore the measuring setup is extended by a network analyzer, in order to evaluate the phase difference between the reference position and the position within the test volume.

\section{Results of the two antenna setup}

The presented two antenna measurement and all done considerations, concerning possible distortions of the DS in TD and the investigation of the pure TEM-mode in TD and FD, are performed.

\subsection{PCC within test volume}

First of all the transmission characteristic of the GTEM1250 for a $100 \mathrm{MHz}$ DS is presented. This type of evaluation and the performance of a damped sinusoidal is already done by Briest ey al. (2015) before. In this paper the existing measurement setup is expanded with a second antenna, working as a reference. Due to this modification a reduction of the signal generator reproducibility and its influence to the PCC is expected.

The PCC is calculated by (1) for 64 measuring positions within in the test volume and is depicted in a heatmap (Fig. 3a), represented by a colorbar. Additional the PCC along the $z^{\prime}$ axis for $x^{\prime}=-7 \mathrm{~cm}$ is presented (Fig. 3b), to get a detailed impression of the PCC characteristics.

Before an analysis of the calculated PCC can be performed, the PCC values have to be ranged. Thus, a PCC of 1 represents a perfect match between the reference and the signal measured in the test volume. In that case the waveguide can be described as a linear time-invariant system for the applied transient signal. Every PCC which deviates from a $\mathrm{PCC}=1$ indicates a distorted signal.

The PCC decreases along the $z^{\prime}$ axis, the direction of the propagated TEM wave, from a maximum PCC of 0.98 to a minimum of 0.92 . This decrease is a indication for an increasing distortion, at least a variation of the DS measured in

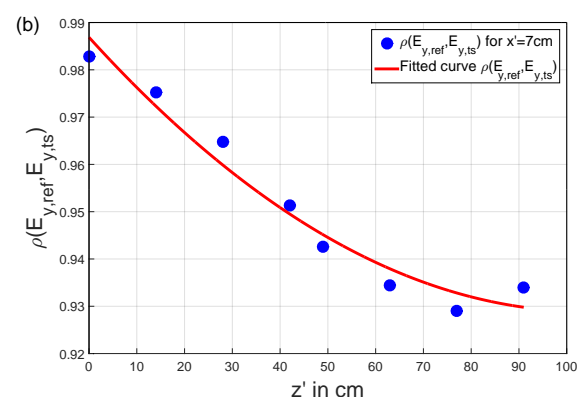

(a) PCC within the test volume for a $100 \mathrm{MHz}$ DS. (b) PCC for

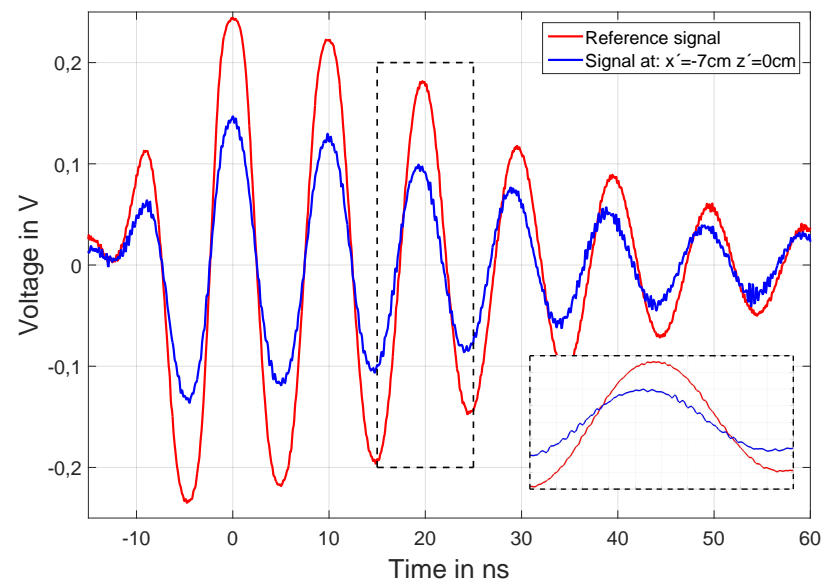

Figure 4. Comparison of $100 \mathrm{MHz}$ DS in TD at $z^{\prime}=0 \mathrm{~cm}$ (Fig. 3) with $\Delta t=0.18 \mathrm{~ns}$.

the test volume from the reference signal. Further inhomogeneous sections within the test volume can be identified. According to Fig. 3a, on the left side of the GTEM1250 the cell door is placed. The measuring positions, for $x^{\prime}=-49 \mathrm{~cm}$, are located close to the cell door. At this position the PCC compared to the PCC at $x^{\prime}=49 \mathrm{~cm}$ is about 0.05 lower, what indicates the influence of the cell door.

The difference between the maximum PCC of 0.98 and the minimum of 0.92 should be discussed in detail. Therefore, the signals within the test volume, with those calculated limit PCC values, are measured in TD and depicted in Fig. $4\left(x^{\prime}=-7 \mathrm{~cm}, z^{\prime}=0 \mathrm{~cm}\right)$ and Fig. $5\left(x^{\prime}=-7 \mathrm{~cm}\right.$, $\left.z^{\prime}=91 \mathrm{~cm}\right)$, each in comparison with the waveform measured at the reference.

In Fig. 4 both signals have a $\Delta t=0.18 \mathrm{~ns}$ delay to each other. In contrast to the signal shown in Fig. 5, where a delay of $\Delta t=1 \mathrm{~ns}$ can be analyzed. In comparison to the reference signal, the measured DS within the test volume is slightly distorted. This distortion is discussed, in reference to present TEM field conditions and a potentially propagation of higher order harmonics in TD and FD, in the following sections. 


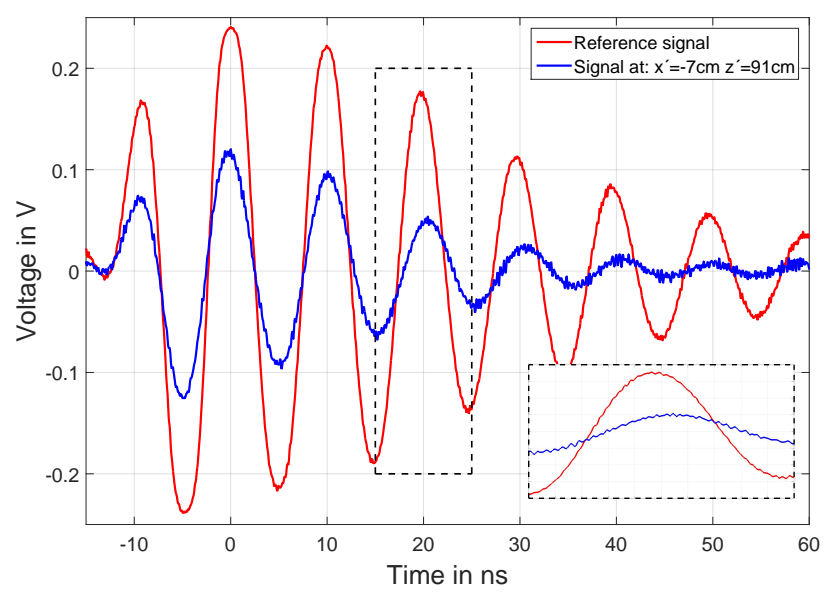

Figure 5. Comparison of $100 \mathrm{MHz}$ DS in TD at $z^{\prime}=91 \mathrm{~cm}$ (Fig. 3) with $\Delta t=1 \mathrm{~ns}$.

So far it is shown, that the PCC is a sensitive method to identify signal distortions in GTEM cells and to qualify a variation of the performed waveform. A threshold value of the PCC, to decide if a waveform is transmitted shape accurate, has to be verified.

\subsection{Discussion of Distorting Effects in TD}

According to the theory of TEM fields (Balanis, 2005), TEM field conditions can be identified by different criteria, mentioned in Sect. 2. Two of these criteria are discussed, by employing the presented measurement setup. In this section the ratio between the primary and secondary $E$-field components in TD is evaluated. As defined before, under TEM conditions the $E$-field only exist in the predominant direction $E_{y}$ within a TEM waveguide, taking into account the required deviations, given by the IEC 61000-20-4.

The distortion of the performed DS is verified under the mentioned criteria. Therefore the $E$-field is measured in three directions $-E_{y}, E_{x}$ and $E_{z}$ - at the reference position of the GTEM1250 and within the test volume, where the distortion of the DS is revealed $\left(x^{\prime}=-7 \mathrm{~cm}, z^{\prime}=91 \mathrm{~cm}\right)$. The comparison of the $E$-field components for the reference position is depicted in Fig. 6. Also the ratio of each secondary component to the primary $E$-field is specified.

The $E_{x}$ component is $16.1 \%$ of $E_{y}$. This value can be explained by a suboptimal probe positioning. Under pure TEMmode conditions at the reference position, a deviation from the z-axis of $\Delta \alpha=9.3^{\circ}$ would correlate to a $E_{x}$ component of $16.1 \%$. For the used probe bracket a minimal rotation $\left(\Delta \alpha \leq 5^{\circ}\right)$ can not be suspended and therefore a minimal $E_{x}$ component exist also under TEM conditions. Due to the spherical wave propagation (Balanis, 2005) also a $E_{z}$ exists at the reference position. The geometry of the TEM waveguide, an angle between the waveguide septum and the floor of $15^{\circ}$, can lead to a $E_{z}$ component of $13 \%$ of $E_{y}$. According to calculations and the investigation of Koch (1998), it

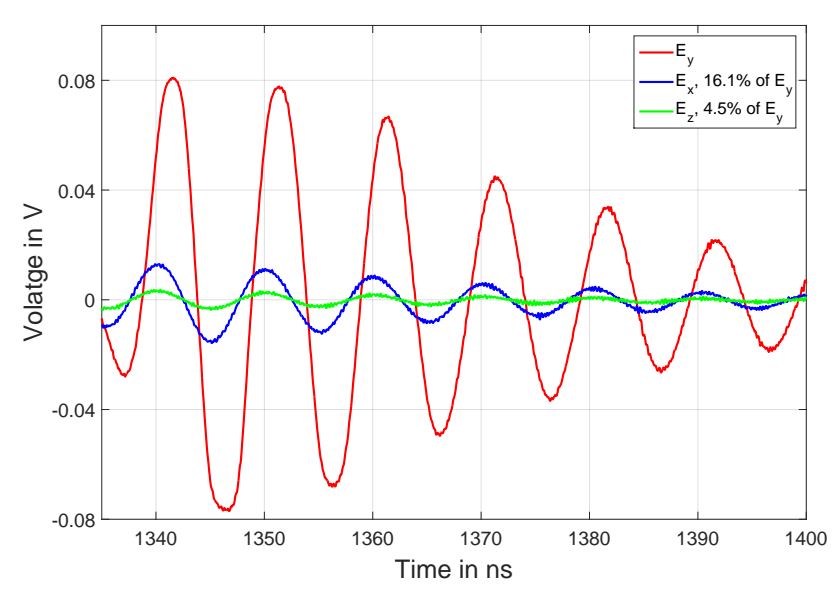

Figure 6. Components of a $100 \mathrm{MHz}$ DS at the reference position.

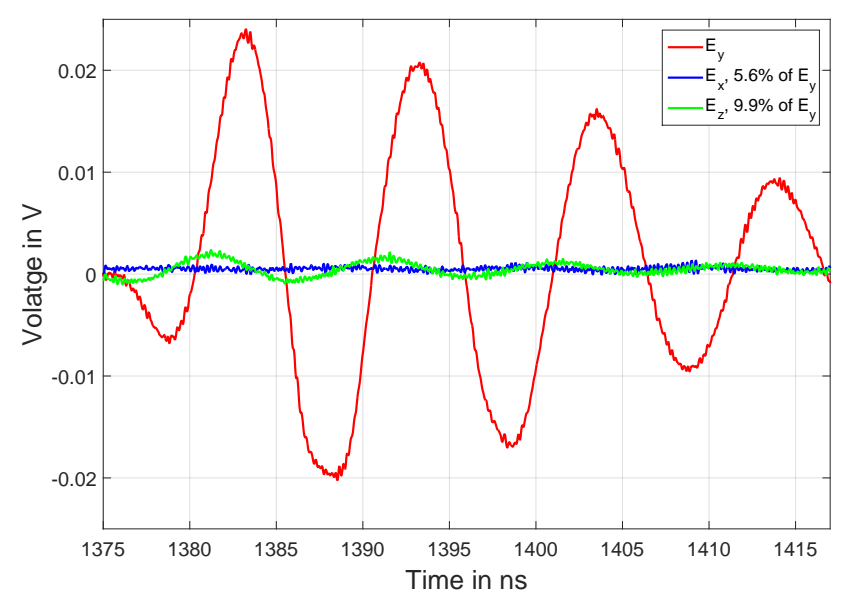

Figure 7. Components of a $100 \mathrm{MHz}$ DS at a septum height of $1 \mathrm{~m}$.

can be assumed that only the pure TEM-mode is present at the reference position.

The $E$-field components within the test volume are compared in Fig. 7. Here a clear increase of the $E_{z}$ component to $\approx 10 \%$ of $E_{y}$ can be registered, what is an explicit variation in comparison to the secondary field component $E_{z}$.

To verify, whether the $E_{z, \mathrm{~s}}$ is increased by a distorting effect of the GTEM1250, further investigations are done.

Due to spherical wave propagation the measured $E$-field - $\left(E_{\mathrm{TEM}}\right)$ under pure TEM conditions - contains a $z$ component. Therefore, the resulting $z$ component of the $E$-field in the test volume $\left(E_{z, \mathrm{~s}}\right)$ can be described as a superposition of $E_{z, \text { TEM }}$ and the distorting effect of the GTEM1250.

According to this theory it should be possible to extract this influence $\eta$ of the GTEM1250 in TD. This can be performed by a subtraction of the $E_{z}$ components between the reference position $E_{z, \mathrm{r}}$ and the measured signal in the test volume $E_{z, \mathrm{~s}}$

$\eta_{E_{z}}=E_{z, \mathrm{~s}}-E_{z, \mathrm{r}}$ 


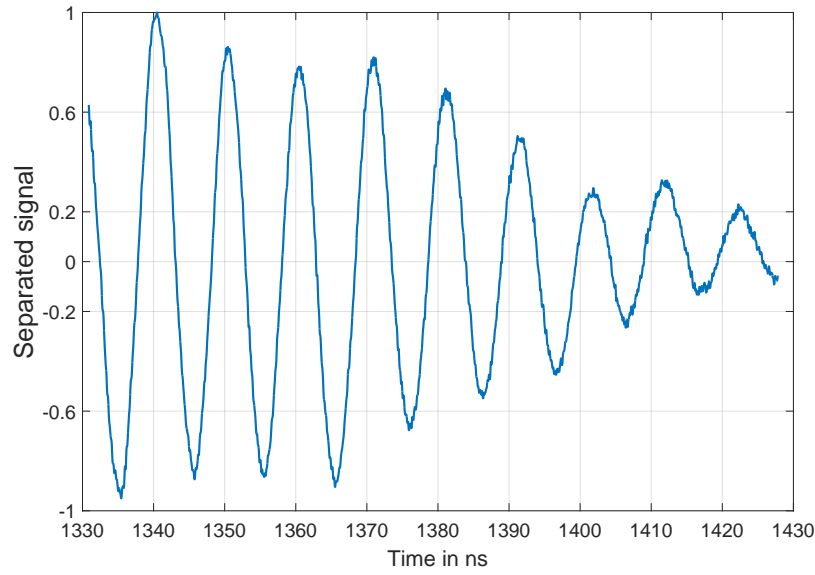

Figure 8. Isolated influence $\eta_{E_{z}}$ to the $E_{z}$ component within the test volume.

First the reference signal amplitude $E_{z, \mathrm{r}}$ has to be normalized $\left(E_{z, \text { norm }}\right)$. This normalisation is done with respect to the difference of the septum-bottom height $\left(h_{\text {septum }}\right)$ between both measurement positions and includes the different field strengths with $E \sim \frac{1}{r}$.

$E_{z, \text { norm }} \approx E_{z, \mathrm{~s}}=E_{z, \mathrm{r}} \times \frac{1}{h_{\text {septum }}}$

The resulting signal $\eta_{E_{z}}$ of the subtraction is shown in Fig. 8.

This oscillating signal can be described as a DS, with a frequency of $98 \mathrm{MHz}$. This frequency is very close to the critical frequency of the GTEM1250 ( $\left.f_{\text {critical }} \approx 97 \mathrm{MHz}\right)$. But it can be eliminated, that this frequency results from the performed DS with a mid band frequency of $100 \mathrm{MHz}$. Compared to the performed DS, the amplitude is constant over 5 periods and can not be described with the typical double exponential envelope. The increased $E_{z}$ component of the measured DS in the test volume (Fig. 5) can be declared.

\subsection{Group velocity delay due to non linearly phase response}

Another criteria to identify distorting effects of TEM waveguides, is the phase response of a propagating wave. A TEM wave propagates with the vacuum speed of light $c_{0}$ (Balanis, 2005). In contrast, higher order modes have a lower propagation speed. Therefore, a measurement of the phase response respectively a measurement of the signal propagation time, between the specified measuring positions (Fig. 1) is performed. The two antenna measurement setup is performed with a network analyzer, which measures the phase response of the propagating wave in means of the scattering parameters ( $S$ parameter).

The $S_{21}$ parameter, measured between the reference position and the feeding point of the cell, and the $S_{31}$ parame- ter, between the test volume $\left(x^{\prime}=-7 \mathrm{~cm}, z^{\prime}=91 \mathrm{~cm}\right.$, Figs. 2 and $3 \mathrm{a})$ and the feeding point of the cell, are measured in a frequency range between $50 \mathrm{MHz}$ and $1 \mathrm{GHz}$. The phase response $\Delta \varphi$ between both measuring points is used to calculate the propagation time in Eq. (4).

$\Delta t=\frac{\Delta \varphi}{360^{\circ} \times f}$

$\Delta \varphi$ is calculated by the subtraction of $\varphi_{S 31}$ and $\varphi_{S 21}$. If the propagation exist only of a TEM-mode $\Delta t$ can be described as constant value of $\frac{d}{c_{0}}$ for the discussed frequencies, where $d$ describes the distance between both measuring positions $(d=2.8 \mathrm{~m})$. Under TEM conditions - propagation speed of $c_{0}$ - the propagation time should have a value of $\Delta t \approx 9.3 \mathrm{~ns}$ (Fig. 9, red line). To identify deviations from $\Delta t \approx 9.3 \mathrm{~ns}-$ TEM conditions - and mark those frequencies, $\Delta t$ is corrected by the constant value of $9.3 \mathrm{~ns}$. This normalized propagation time $\Delta t^{\prime}$ is depicted in Fig. 9 (blue line). For used network analyzer a phase noise of $\Delta \varphi= \pm 0.11^{\circ}{ }^{1}$ is denoted, which has to be taken into account. The phase noise results in an uncertainty according to Eq. (5).

$U_{\Delta \varphi}=2 \times \frac{0.11^{\circ}}{\sqrt{3}}$

The uncertainty $U_{\Delta \varphi}$ affects to an uncertainty $U_{\Delta t}$ of the signal propagation time according to Eq. (4) (Fig. 9, green line)

$U_{\Delta t}=\frac{U_{\Delta \varphi}}{360^{\circ} \times f}$.

Above a frequency of $200 \mathrm{MHz}$ the shown propagation time is $\Delta t^{\prime} \approx 0 \mathrm{~s}$. At each frequency where $\Delta t^{\prime} \approx 0 \mathrm{~s}$ the TEM-mode is dominant and the speed of wave propagation is $c_{0}$. At frequencies with a deviation from this value the signal propagation speed is $\neq c_{0}$ and the pure TEM-mode does not exist.

In Sect. 3.2 the first higher order mode at a frequency of $97 \mathrm{MHz}$ is verified by the secondary $E$-field components. Here, a normalized signal propagation time of $\Delta t^{\prime} \approx 0.83 \mathrm{~ns}$ is calculated and is significantly higher than $\Delta t^{\prime}$ at frequencies above $200 \mathrm{MHz}$. According to the theory of higher order mode propagation, the first higher order mode can be identified by its significant lower propagation time at $f \approx 97 \mathrm{MHz}$.

$\Delta t_{f<97 \mathrm{MHz}}^{\prime}$ are significantly smaller than $\Delta t^{\prime}$ at $97 \mathrm{MHz}$. In contrast, the expanded uncertainty of the measured phase increases around these frequencies, which has to be taken into account.

\footnotetext{
${ }^{1}$ Keysight N5222A with 85052B Calibration Kit-Specification Table 4a: http://literature.cdn.keysight.com/litweb/pdf/N5221-90001.pdf? id $=2080065$
} 


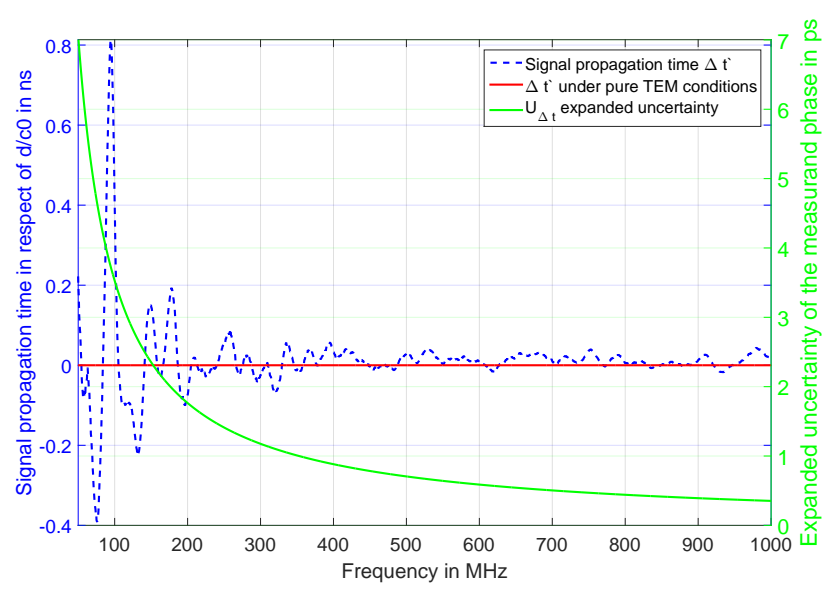

Figure 9. $U_{\Delta t}$ and $\Delta t^{\prime}$, with an offset of $\frac{d}{c_{0}}$.

\section{Conclusions}

This paper discusses whether the double exponential pulse describes the transmission quality of a TEM waveguide for arbitrary transient signals sufficiently. Therefore, the transmission characteristic is evaluated in time and frequency domain. In TD it can been shown that specific transient signals with frequencies in the spectrum of the double exponential pulse are transmitted not shape inherently.

Based on investigations done in Briest ey al. (2015), an expanding two antenna measurement method is presented to verify the transmission quality of any transient signals in TEM waveguides. The reduction of the signal generator reproducibility and its influence to the PCC is a substantial advantage compared to previous measurement setup. Further, investigations in FD can be performed to discuss the transmission characteristics of the TEM waveguide in detail. It is based on two measuring positions, where a reference signal and signals within the test volume of the waveguide are measured. The PCC of the measured signals is calculated and presented by a heatmap. It allows a quick evaluation and characterization of the transmission quality of the TEM waveguide.

Furthermore an investigation of distorting effects of the waveguide is performed. Two significant criteria of the theoretically present TEM-mode are discussed. Contrary to a pure TEM-mode excitation, secondary $E$-field components can be measured in the direction of propagation. The other typical criteria of a non pure TEM wave is its reduced propagation speed in comparison to the speed of light $c_{0}$ for a pure TEM-mode propagation. Therefore, an analysis which is also based on the two antenna measurement setup is performed in FD in order to evaluate the phase response of the GTEM 1250 cell. From the measured phase response, the group velocity can be calculated and discussed. It can be shown that the group velocity at the critical frequency of the GTEM 1250 shows a significant difference to the group velocity $c_{0}$ of the pure TEM-mode.

It is shown that the double exponential pulse from the IEC 61000-4-20 only validates the TEM waveguide for a double exponential pulse. Other pulses with their significant spectrum within the spectrum of the double exponential pulse, may not be transmitted perfectly. In summary, the waveguide transmission quality has to be evaluated for every different transient signal which are intended to use for emission or immunity tests.

Acknowledgements. We acknowledge support by Deutsche Forschungsgemeinschaft and Open Access Publishing Fund of Leibniz Universität Hannover. The results shown in this paper were partly produced with the support of the Bundeswehr Research Institute for Protective Technologies, NBC-Protection in Munster, Germany. Contract Number E/E590/CF148

The publication of this article was funded by the open-access fund of Leibniz Universität Hannover.

Edited by: F. Gronwald

Reviewed by: three anonymous referees

\section{References}

Balanis, C. A.: Antenna theory: analysis and design, 3. Edn., WileyInterscience, Hoboken, NJ, 2005.

Briest, N., Garbe, H., and Potthast, S.: Transmission characteristics of a TEM waveguide for transient signals by the use of a damped sinusoidal, Adv. Radio Sci., 13, 175-179, doi:10.5194/ars-13175-2015, 2015.

Hamann, D. and Garbe, H.: Enhanced Estimates of Field Distribution's Uncertainty Contribution for TEM Waveguide, 13th IEEE Int. Symposium on Electromagnetic Compatibility, Raleigh, USA, 3-8 August, 2014

Kärst, J. P.: Qualifikation beladener TEM-Wellenleiter, PhD-Thesis, GEM, Leibniz University, Hannover, Germany, 2002

Koch, M.: Analytische Feldberechnung in TEM-Zellen, PhDThesis, GEM, Leibniz University, Hannover, Germany, 1998.

Kölling, C., Zamow, D., and Garbe H.: A Correlation Method to Extend the IEC 61000-4-20 for UWB Measurements, 10th IEEE Int. Symposium on Electromagnetic Compatibility, York, UK, 26-30 September, 2011.

Kortke, A. and Mann, W.: Near field scanning with optoelectronic E-field probes, 3rd European Conference on Antennas and Propagation, Berlin, Germany, 23-27 March, 2009.

Radasky, W. A., Smith, K. S., Hansen, D., and Ristau, D.: Calculations and measurements of fast EM pulses in the GTEM cell, International Symposium on Electromagnetic Compatibility, Symposium Record, Santa Clara, USA, 19-23 August, 1996.

TESEQ AG: Definition of Measurement Points for Field uniformity, GTEM Cells, http://www.teseq.com/products/downloads/ brochure/GTEM_Cells.pdf, 2014. 